\title{
Experimental Analysis of Small Diameter Brush Seals and Comparisons With Theoretical Predictions
}

\author{
Lilas Deville \\ PPRIME Institute, UPR CNRS 3346, Université de Poitiers, ENSMA ISAE, Chasseneuil Futuroscope 86962, France \\ Mihaï Arghir \\ PPRIME Institute, UPR CNRS 3346, Université de Poitiers, ENSMA ISAE, Chasseneuil Futuroscope 86962, France
}

The paper presents the experimental results obtained for brush seals of $38 \mathrm{~mm}$ diameter operating with air at pressure differences up to 7 bars and rotation frequencies up to $500 \mathrm{~Hz}$. The seals had bristles of $70 \mathrm{~lm}$ diameter, made of Haynes 25. Seals with two radial interferences $(0$ and $100 \mathrm{~lm})$ between the brush and the rotor were tested. The pre-sented running in procedure underlines the influence of the initial wear on the brush tem-peratures. The test results consisted of leakage mass flow rates. The temperatures of a limited number of points on the brush and on the rotor were also recorded. The results confirmed the important impact of the radial interference on the leakage. The test data were further confronted with theoretical predictions obtained with an original model. The model considers the brush as a deformable porous medium. Its local porosity and permeability are obtained from a fluid-structure interaction between the bristle pack and the leakage flow. The comparisons showed nearly close values of the mass flow rates. The differences between experimental and theoretical predictions are considered to be due to an underestimation of the porosity because the model neglects the friction forces between bristles and between the bristles and the rotor.

Keywords: brush seals, experimental analysis, leakage mass flow rate

\section{Introduction}

Brush seals represent the most widely used technology of compliant sealing of the moment. The technology was introduced almost three decades ago and received increasing attention, because it proved to be a very good replacement for the labyrinth seals traditionally used in rotating machinery. Labyrinth seals have relatively important radial clearances in order to accommodate the dynamic radial displacements of the rotor. In brush seals, the radial clearance is completely obstructed by a porous media made of strongly compacted bristles (Fig. 1). This leads to small porosities and therefore to a high flow resistance.

Compared to labyrinth seals, the leakage flow is at least one order of magnitude lower. Moreover, the width of the brush seals is much smaller than the length of labyrinth seals. The brush generally interferes with the rotor surface, i.e., the bristles are longer than the radial clearance. However, due to its compliance, the brush accommodates radial displacements of the rotor. Most brush seals have bristles made of super alloys with diameters of the order of $70-150 \mu \mathrm{m}$, or of aramid or carbon fibers with much smaller diameters. The bristles are inclined in the direction of rotation with a lay angle generally comprised between $30 \mathrm{deg}$ and $60 \mathrm{deg}$. This allows an easy bending of the bristles. The interference of the bristles with the rotor surface is a key point, because the heat generated in the contact might have damaging effects.

The aforementioned characteristics of brush seals were proved on tests performed on rotating machinery prototypes or retrofits. The collected experimental information supported the subsequent development of theoretical models. In 1991, Hendricks et al. [1] introduced a theoretical model based on empirical correlation for the leakage flow. The bristle deformation was computed from a beam analysis. Chupp et al. [2,3] proposed an approach relying on a friction factor coefficient that depended on the width of the brush. These models use simple physical arguments, and therefore, they rely strongly on experimental results. Braun and Kudriavtsev [4] performed computational fluid dynamics (CFD) analyses of the flow inside the brush. They analyzed the two-dimensional flow in a regular arrangement of closely packed cylinders simulating the bristles. Their analysis enabled a good visualization of flow details but remained limited being only two dimensional.

Another category of more recent theoretical models considers the brush as a porous medium [5-10]. These methods can be considered as extensions of the approach used by Chupp et al. [2,3].

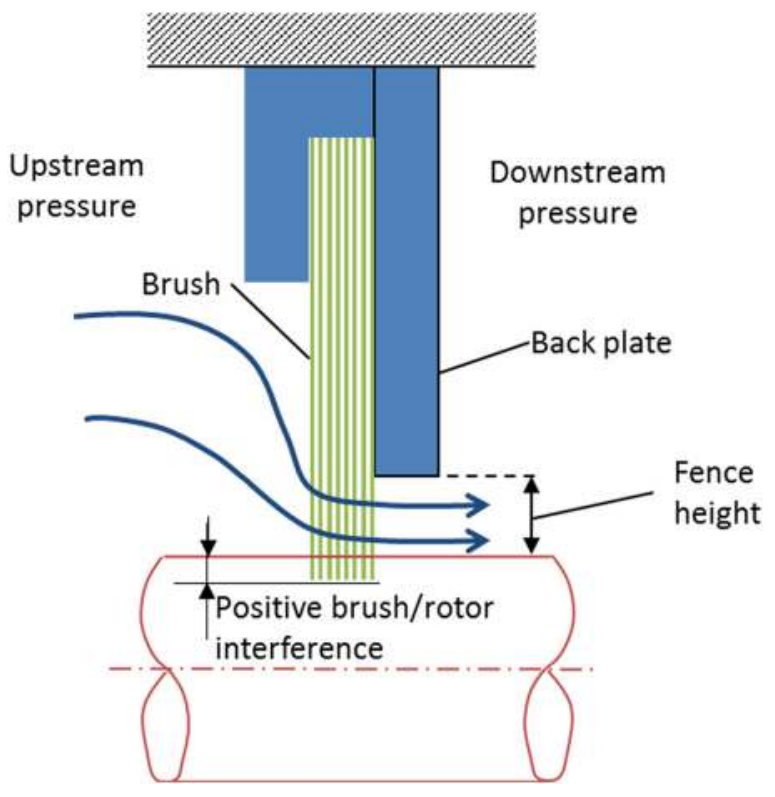

Fig. 1 Schematic view of the brush seal 


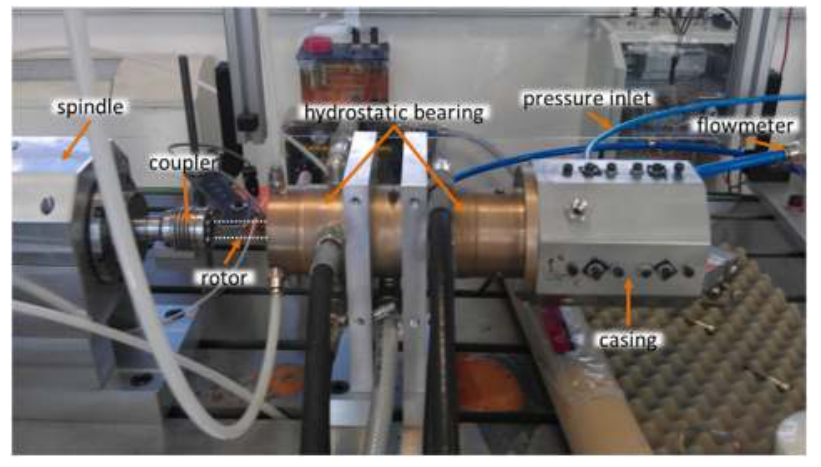

Fig. 2 Test rig

However, they also require experimental information for correlating the porosity and the permeability. This experimental information generally comes from tests performed by the authors under specific operating conditions as for example in Refs. [6,8] and [10]. The applicability of the porous media model was theoretically and experimentally confirmed by other experiments/tests $[11,12]$.

Until now, Lelli et al. [13] presented the most elaborate brush seal model. The model is similar to the approach used in Ref. [9] and is based on an iterative fluid-structure interaction between the bristles modeled as elastic bodies and the brush flow calculated from a CFD approach. The model was validated by comparing the estimated torque with experimental data but flow rates were not reported.

Recently, the present authors have introduced a similar model that assimilates the brush with a porous, deformable medium [14]. As in the model of Guardino and Chew [9], the bristles deform under the effect of pressure forces, due to the interference with the rotor and due to the contact with the back plate. It results a porous medium with spatially varying porosity. The permeability is computed from the analogy with the pressure drop in a duct. This approach was first introduced by Prostler [8]. He considered a brush with a constant porosity and Ergun's relation [15] for the dimensionless pressure drop in a pipe. In the theoretical model introduced by the present authors, Ergun's relation was replaced with the pressure drops calculated by a CFD approach in each of the three principal directions. Different computational domains were considered for each principal direction and realistic spacing between the bristles was used. The mass flow rate can be predicted without an a priori knowledge of the brush seal porosity and permeability and independently of any experimental data. The model was validated by comparisons with the experimental data of Bailey and Long [16], which were the only one in the literature containing the details that the model needed. Other researchers used the same experimental results for validating brush seals theoretical models [9], because it documents well the geometric characteristics of the brush.

This study presents the results of the experimental program developed by the authors and aimed to provide data for enforcing the validation of their theoretical model. The tested brush seals were of small diameter $(38 \mathrm{~mm})$ and were tested for different feeding pressures and rotor speeds. In the following, the paper presents the test rig, the main steps of the test campaigns, the results obtained for steady operating conditions, ${ }^{1}$ and comparisons with the theoretical model introduced in our paper [14].

\section{The Test Rig and the Tested Seals}

The test rig depicted in Fig. 2 is composed of a rotor guided by two identical hydrostatic bearings fed with water at 30 bar. An electric spindle at its left-hand end drives the rotor and a test casing is overhung mounted at its right-hand side end. The brush

\footnotetext{
${ }^{1}$ Steady operating conditions indicate the absence of any imposed rotor vibrations. The results consist of the leakage versus pressure drops and temperatures.
}

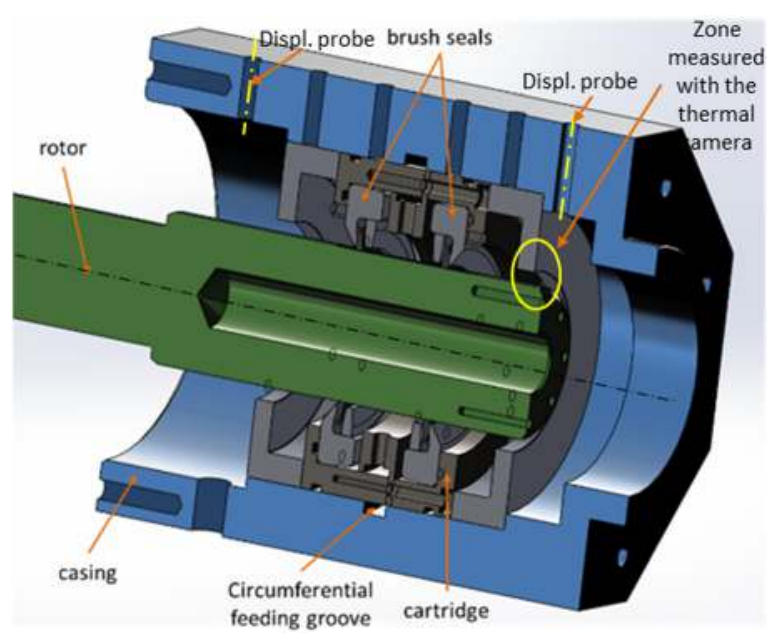

Fig. 3 Test cartridge installed in the casing

seals are tested by pairs, mounted in a cartridge, in back-to-back arrangement, as shown in Fig. 3. The cartridge is installed in the casing mounted on the test rig. Pressurized air at ambient temperature is fed into the cartridge via a circumferential groove located midway between the two brush seals. This is very similar to a buffer seal arrangement used in turbo engines and compressors. The two sides of the cartridge are at ambient (atmospheric) pressure, so both brush seals are subject to the same feeding pressure and pressure drop.

The mass flow rate for the two seals, the inlet pressure, the rotation speed, and the rotor displacements are measured on the test rig. The measurement accuracy is \pm 0.2 bar for pressure, $\pm 10 \mathrm{~Hz}$ for rotation frequency, and $\pm 0.2 \mathrm{~g} / \mathrm{s}$ for the mass flow rate. The temperature at the interface between the rotor and the right-hand side brush seal is monitored using a thermal camera (Fig. 4). This measure provides only an indicative temperature, because the emissivity of the brush is not known.

The tested brush seals were of the kind described in Ref. [17] and their characteristics are given in Table 1. As shown in Fig. 1, they are mounted on the rotor either with a $100 \mu \mathrm{m}$ radial interference or without interference, i.e., an inline configuration with no cold clearance. The interference is obtained by using two rotors with appropriately modified diameters verified by metrology.

The static eccentricity of the rotor was not measured but was supposed to be negligible, because the hydrostatic bearings guiding the rotor are fed with pressurized water at 30 bar, so their radial stiffness is very large compared to brush seals. An analytic estimation of the rotor growth for a rotation frequency of $500 \mathrm{~Hz}$ gives a $3.6 \mu \mathrm{m}$ increase of the shaft radius.

\section{Running in of the Seals}

The brush seals were first submitted to a running in procedure. The procedure consists of tests performed by combining two

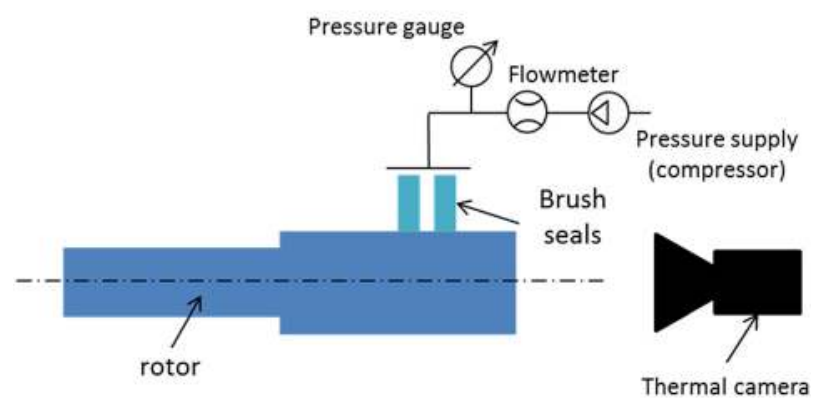

Fig. 4 Instrumentation of the test rig 
Table 1 Brush seals data

\begin{tabular}{llll}
\hline \hline Bristle characteristics & & Seal assembly \\
\hline Material & Haynes 25 & Rotor diameter & $38.355 \mathrm{~mm}$ \\
Diameter & $0.07 \mathrm{~mm}$ & Interference & $0.1 \mathrm{~mm}$ or 0 \\
Lay angle & $45 \mathrm{deg}$ & Fence height & $0.8 \mathrm{~mm}$ \\
Total length & $9.9 \mathrm{~mm}$ & Bristle density & $100 \mathrm{bristles} / \mathrm{mm}$ of circumference \\
Free radial bristle length & $5.5 \mathrm{~mm}$ & Number of bristles in axial direction & 7 or 8 \\
\hline \hline
\end{tabular}

Table 2 Magnitude of the nonfiltered temperature variation for each running in test

\begin{tabular}{lccccr}
\hline \hline & \multicolumn{2}{c}{$\Delta T$ brush } & & \multicolumn{2}{c}{$\Delta T$ contact } \\
\cline { 2 - 3 } \cline { 5 - 6 } Running in campaign & 1st & 2nd & & 1st & 2nd \\
\hline $100 \mathrm{~Hz} \mathrm{2} \mathrm{bars}$ & 12.23 & 0.8 & 30.88 & 3.14 \\
$100 \mathrm{~Hz} 4$ bars & 2.21 & 1.11 & 21.67 & 3.81 \\
$400 \mathrm{~Hz} 2$ bars & 9.31 & 7.56 & & 21.46 & 8.93 \\
$400 \mathrm{~Hz} 4$ bars & 12.3 & 7.97 & & 16.32 & 13.95 \\
\hline \hline
\end{tabular}

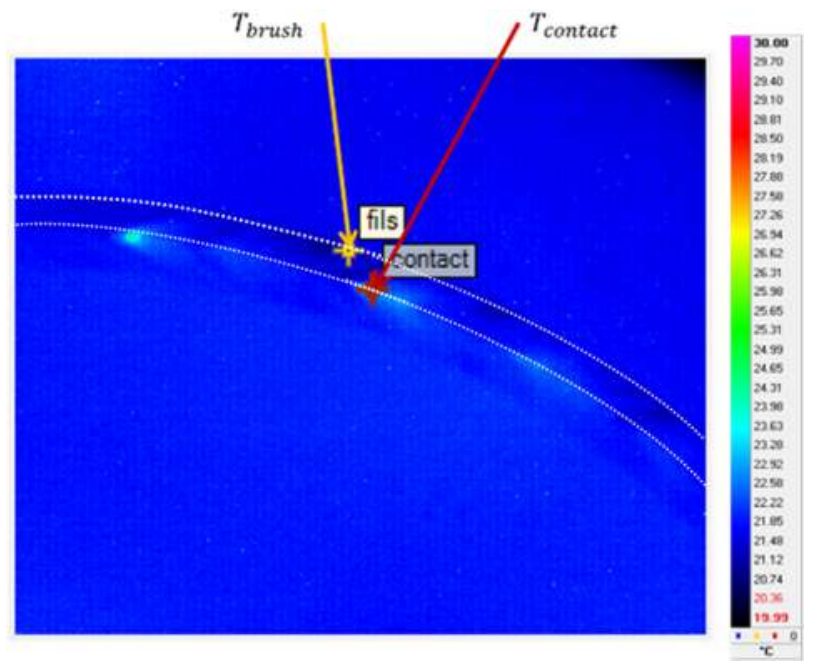

Fig. 5 Frame of the thermal camera and measuring points

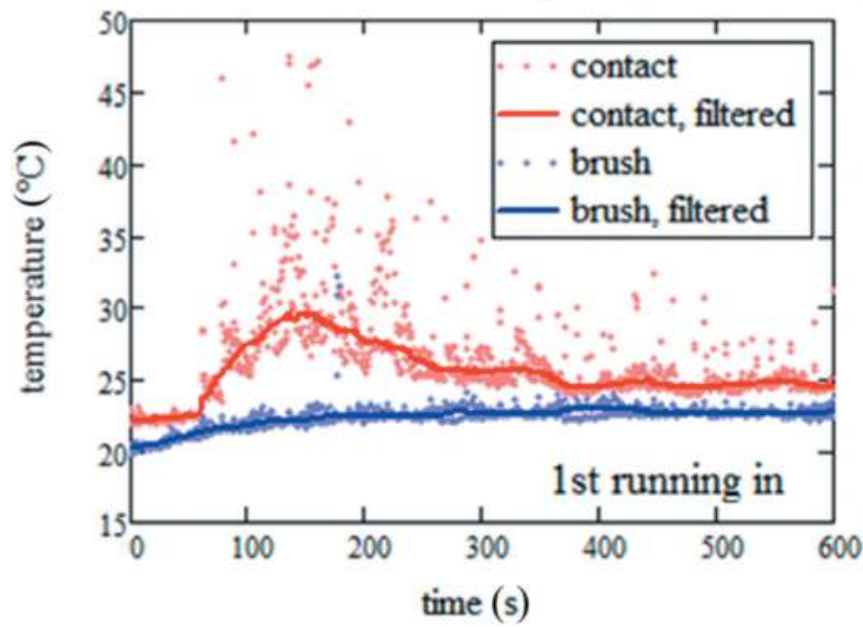

rotation speeds $(100 \mathrm{~Hz}$ and $400 \mathrm{~Hz})$ and two feeding pressures ( 2 bar and 4 bar), i.e., four operating conditions. Each test was carried out for $10 \mathrm{~min}$ and was performed twice. The tests were performed with $100 \mu \mathrm{m}$ radial interference and their order is indicated in the first column of Table 2, from top to bottom. The temperature variations of a point on the brush and of a point at the brush-rotor contact shown in Fig. 5 were used to assert the effectiveness of the running in of the seals. Figures 6 and 7 present the variation of the temperatures (unfiltered, sampled every $1 \mathrm{~s}$ and filtered with a median filter) during the first and second running in for two extreme operating conditions: $100 \mathrm{~Hz}$ and 2 bars and $400 \mathrm{~Hz}$ and 4 bars. It should be underlined that because the emissivity of the brush is not known (it was supposed equal to unity), the absolute temperatures are, therefore, less significant than temperature variations. For the first running in tests, the contact temperature has sudden peaks that may correspond to a change in the rub situation caused by a change in the bristle formation during first time operation. These peaks have a much lower amplitude or are even absent during the second running in tests. Furthermore, the evolution of the temperature shows variations of lower magnitude and of lower dispersion during the second running in tests (Table 2). It was, thus, concluded that the running in of the seals was effective and enabled the homogenization of the bristle pack by equalizing the length of the bristles.

\section{Test Campaign}

Tests were performed at constant rotational frequencies ranging from $100 \mathrm{~Hz}$ to $500 \mathrm{~Hz}$, with $100 \mathrm{~Hz}$ increments. The feeding pressure was progressively increased with 1 bar increments until the mass flow meter became saturated. This occurred close to 7 bars for the $100 \mu \mathrm{m}$ radial interference and close to 4 bars for the cases without interference. The feeding pressure was then progressively decreased, in order to highlight the hysteresis phenomenon characteristic for brush seals. Each test at constant speed lasted approximately $3 \mathrm{~min}$ and was performed twice. The cumulated duration of a complete test was approximately $30 \mathrm{~min}$. The mass flow rate and the upstream pressure were measured as well as the

Fig. 6 Temperature variation during the two running in at $100 \mathrm{~Hz}$ and 2 bars of pressure drop 

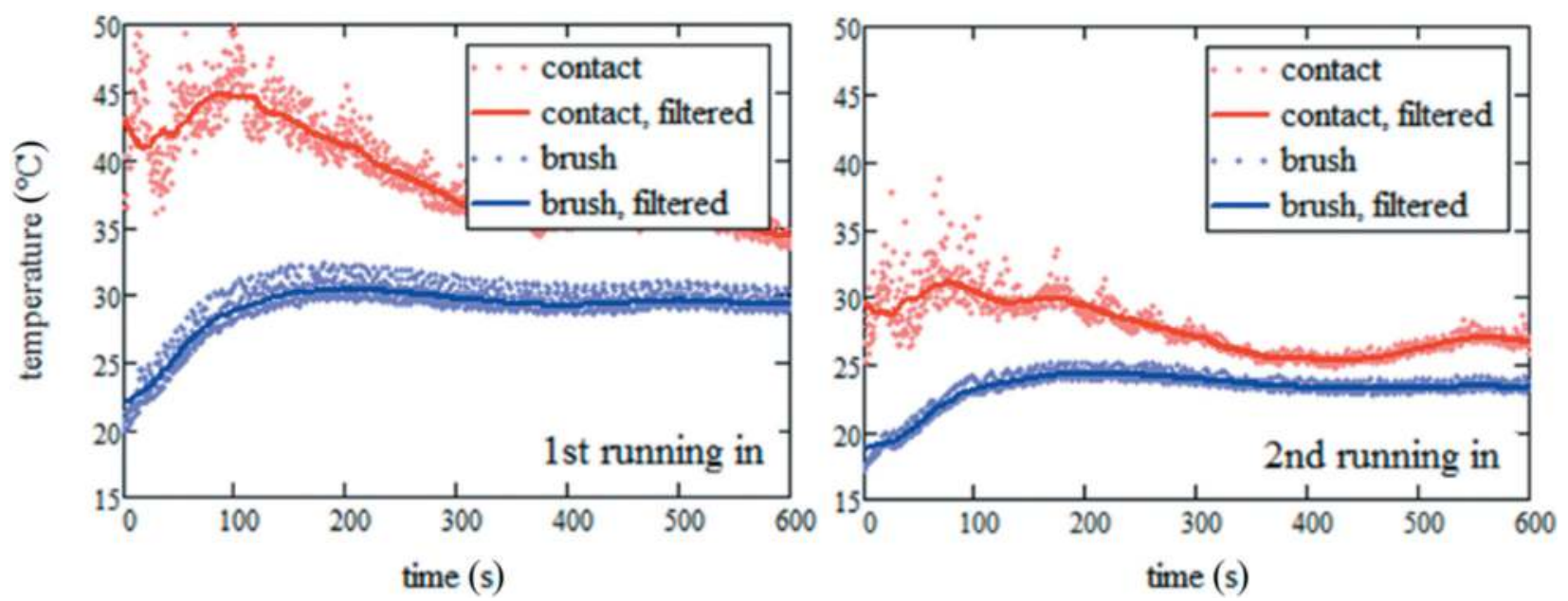

Fig. 7 Temperature variation during the two running in at $400 \mathrm{~Hz}$ and 4 bars of pressure drop
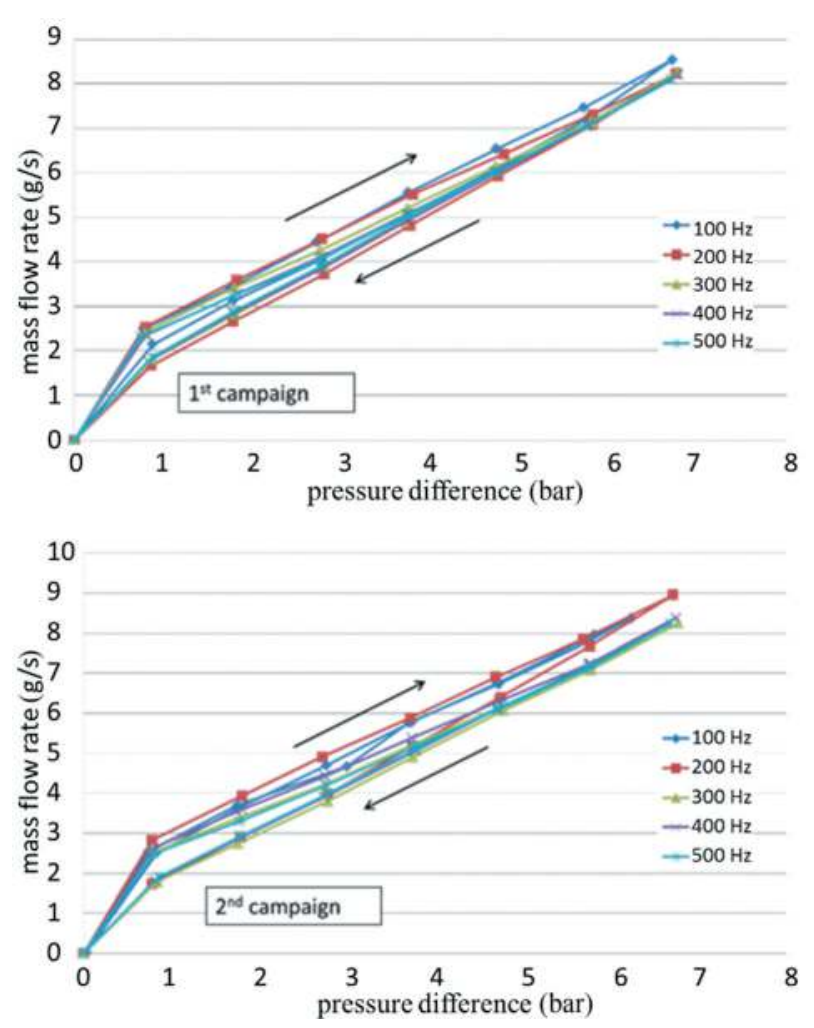

Fig. 8 Mass flow rate measured for two seals mounted with $100 \mu \mathrm{m}$ radial interference versus the pressure drop and for different rotation frequencies

temperature of the brush seal situated at the right-hand side of the cartridge shown on the Fig. 4.

4.1 Leakage Flow. The measured mass flow rate for two back-to-back brush seals mounted with $100 \mu \mathrm{m}$ radial interference is plotted in Fig. 8. The results show a hysteresis phenomenon indicated by arrows. This is characteristic for brush seals and it occurs due to the friction forces acting between the bristles of the brush. While the pressure is increasing, the bristles are progressively pushed against each other and against the backing plate and become compacted. Because of the friction force, when the pressure decreases, the bristles are not free to regain their initial position. For the same value of the pressure difference, the compactness of the brush is then higher during pressure decrease than during pressure increase (i.e., its porosity is smaller), resulting in a lower mass flow rate.

There is also a trend in the experimental results suggesting that the mass flow rate does not tend linearly to zero at zero pressure gradient. This is explained by the fact that the increase of the leakage mass flow rate with the pressure difference is very rapid for small values of this latter, because the bristles are not compacted yet. It is only with the increase of the pressure difference that the bristles will make a compact brush and the leakage mass flow rate will increase less sharply.

Figure 9 depicts the same mass flow rates versus the rotation speed for different pressure drops, but the values are averaged between the two series of tests. The figure shows that the mass flow rates are almost constant with the rotation speed and the results measured at lower rotational frequencies $(100$ and $200 \mathrm{~Hz})$ are slightly higher. The explanation is the fact that the test rig behaves as a dynamic system consisting of the rotor, the two hydrostatic bearings, and the brush seals. Its resonant frequency is located between 150 and $175 \mathrm{~Hz}$. Due to the residual unbalance of the rotor, the dynamic displacements measured near these frequencies are larger than for the rest of rotation frequencies. Figure 10 depicts the rotor dynamic displacements that decrease with increasing rotation frequency from $100 \mathrm{~Hz}$ to $500 \mathrm{~Hz}$. These dynamic displacements may lead to the loss of interference between the brush and the rotor and to a subsequent increase of the mass flow rate.

Figure 11 depicts the mass flow rate measured for two brush seals mounted without radial interference. As for the brush seals

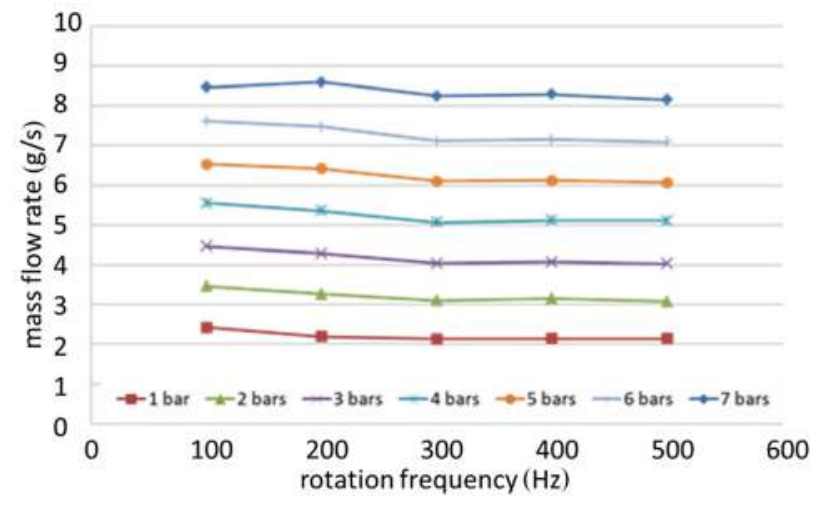

Fig. 9 Variation of the averaged mass flow rates for two seals mounted with $100 \mu \mathrm{m}$ radial interference versus the rotation speed and for different pressure drops 


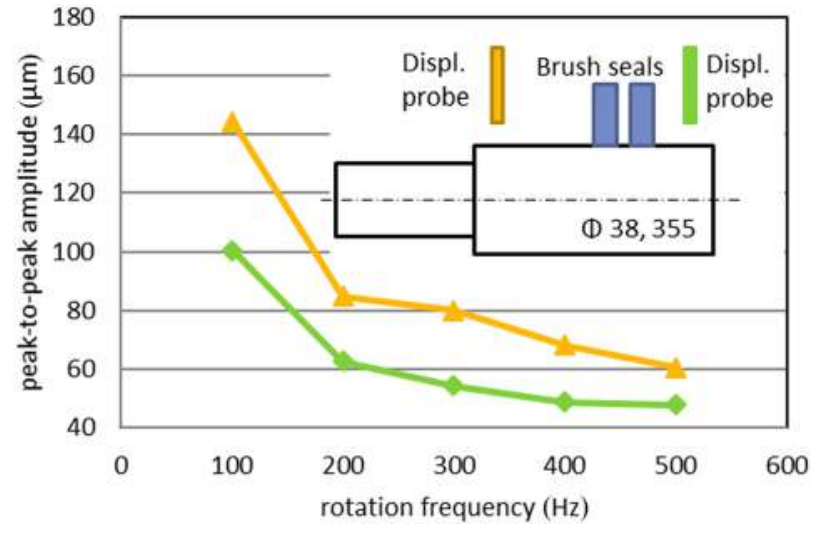

Fig. 10 Amplitude of rotor dynamic displacements for brush seals with $100 \mu \mathrm{m}$ interference
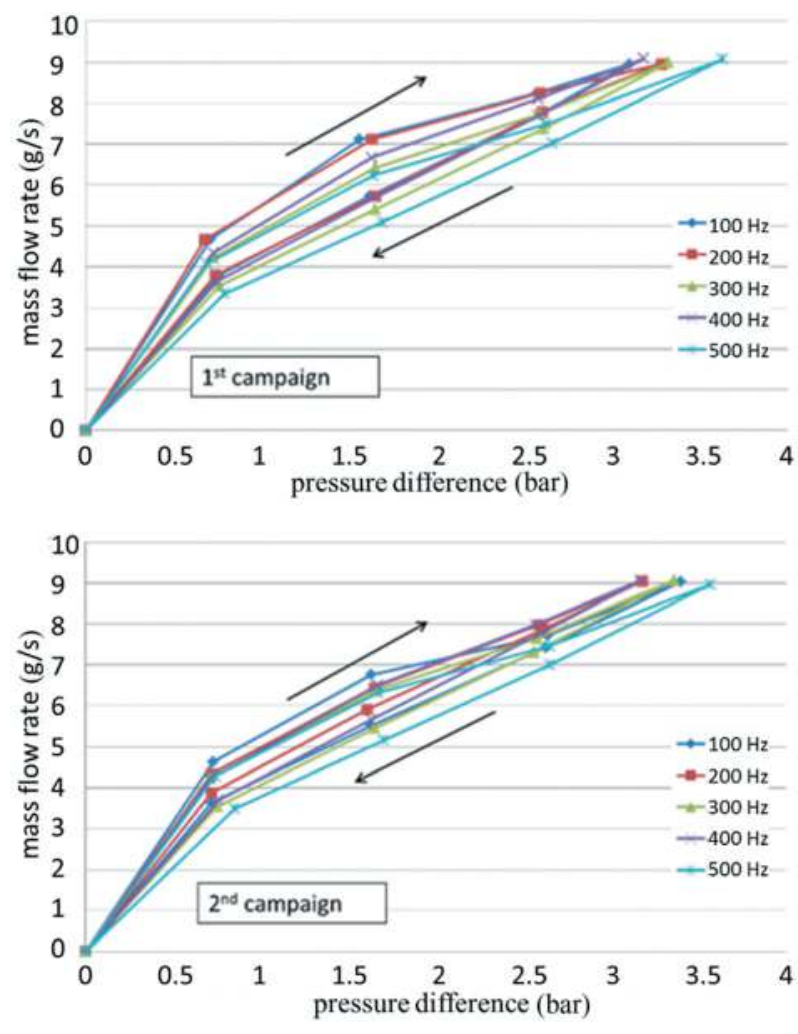

Fig. 11 Mass flow rate measured for two seals mounted without interference versus the pressure drop and for different rotation speeds

mounted with radial interference, the results show a hysteresis phenomenon indicated by arrows. The difference between the mass flow rate measured during pressure increase and pressure decrease is more pronounced than in the case with radial interference. This is because at low-pressure differences, the bristles are just slightly in contact with the rotor. As the pressure is increased, the brush is not only compacted against the backing plate (as in cases with interference) but also pressed against the rotor by the so-called "blow-down effect." The combination of these two effects increases the compactness of the brush, hence the more pronounced hysteresis phenomenon. Figure 12 shows that the rotational speed has a limited influence on the mass flow rate, like in the previous case. Again, the mass flow rates are slightly higher for $100 \mathrm{~Hz}$ and $200 \mathrm{~Hz}$ rotation speeds, which is due to the resonance frequency of the test rig.

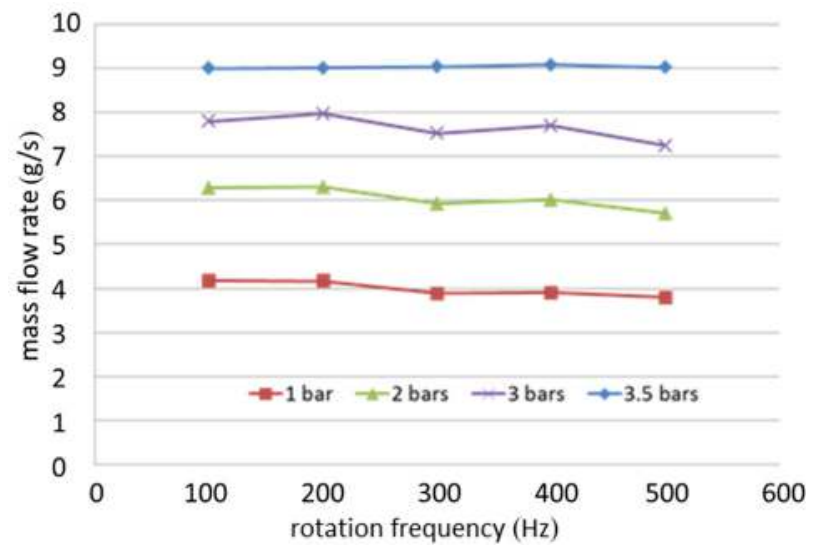

Fig. 12 Variation of the averaged mass flow rates for two seals mounted without interference versus the rotation speed and for different pressure drops

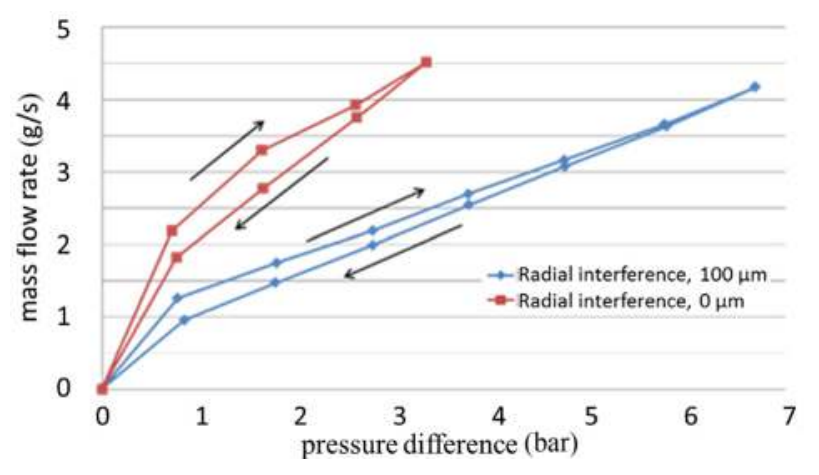

Fig. 13 Comparison between the averaged mass flow rates of a single seal for the two tested interferences

Figure 13 compares the mass flow rates obtained with and without radial interference. The depicted results are the mass flow rates for a single seal obtained by assuming that the two brush seals have identical leakages. In order to enable a clear comparison, the mass flow rates were averaged between all rotation speeds. It can be seen that the brush seal without interference has a mass leakage flow $80 \%$ higher than the seal with $100 \mu \mathrm{m}$ radial interference.

In terms of the discharge coefficient of an equivalent orifice with an area proportional to the rotor circumference and the fence height (Table 1), the highest values of the mass flow rate depicted in Fig. 13 correspond to 0.024 and 0.04 for the seal with and without interference, respectively.

4.2 Temperature. During the tests, the temperature of righthand side seal is monitored using the thermal camera as shown in Fig. 4. Small clips of $10 \mathrm{~s}$ are recorded for each stabilized operating condition. A frame of one of these clips is shown in Fig. 14. The temperature is tracked at four distinctive points: on the brush, on a point approximately located at the brush/rotor contact, on the rotor and on the back plate of the brush seal. The point on the brush was located as close as possible from the midheight of the fence. It is estimated that the points on the rotor and on the back plate are located $10 \pm 2 \mathrm{~mm}$ from the contact line. The temperatures are then averaged and plotted on Figs. 15-17. These figures depict the temperatures (left-hand side scale) and the pressure differences (right-hand side scale) versus the test number. This plotting method was adopted in order to highlight the correlation between the stabilized temperatures and variations of the pressure difference at constant rotation speed. 


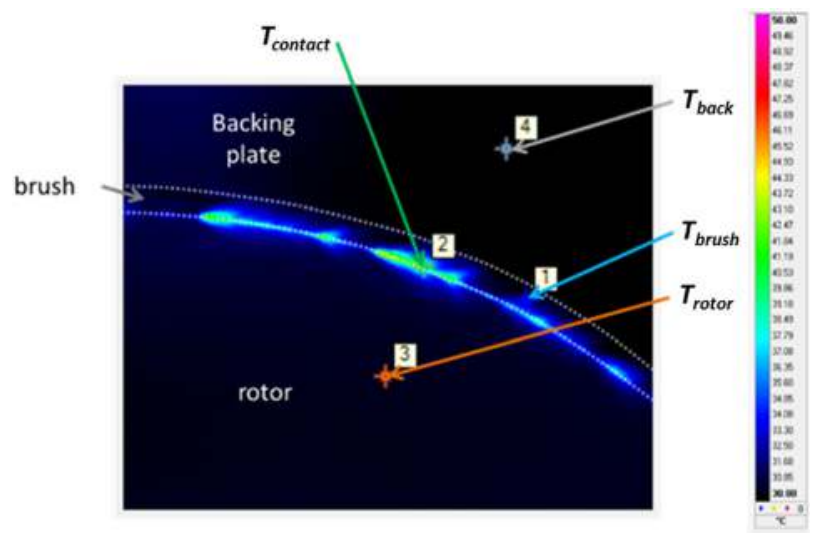

Fig. 14 Temperature measurements on the video clips given by the thermal camera
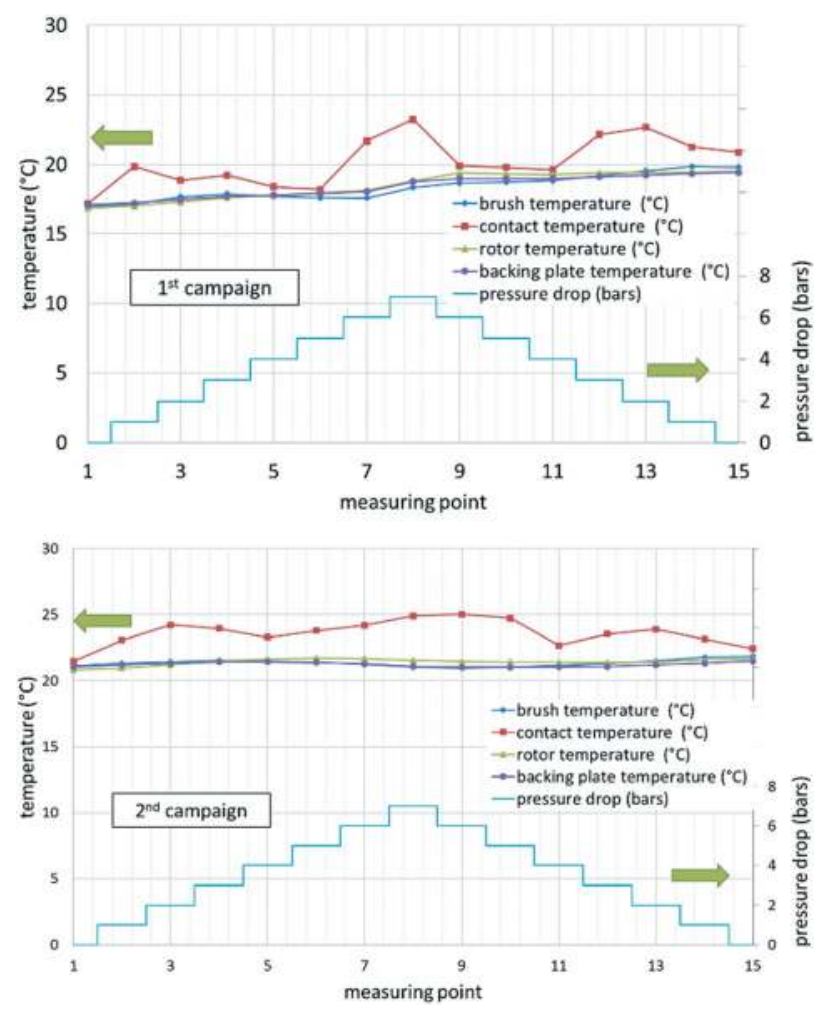

Fig. 15 Temperature variations for $100 \mathrm{~Hz}$ rotation speed (seal with $100 \mu \mathrm{m}$ radial interference)

Figures 15 and 16 depict the temperature variations for the seal with $100 \mu \mathrm{m}$ radial interference. The temperatures of the brush, rotor, and backing plate are very close. The contact temperatures are obviously higher due to the heat generated by the friction of the bristles on the rotor. As normally, the maximum temperatures measured for $500 \mathrm{~Hz}$ rotation speed are larger than for $100 \mathrm{~Hz}$. The contact temperatures have diminished between the first and the second test campaign. This shows that due to wear, the interference of the brush with the rotor decreased slightly, and therefore, the bristles/rotor contact forces and the heat generated by friction diminished.

The temperature of the brush has similar variations for all tests depicted in Figs. 15 and 16. At the beginning of the test, the brush temperature rises slowly as the energy dissipated by contact friction is conducted into the brush. The temperature continues to increase up to a value of the pressure difference that depends on
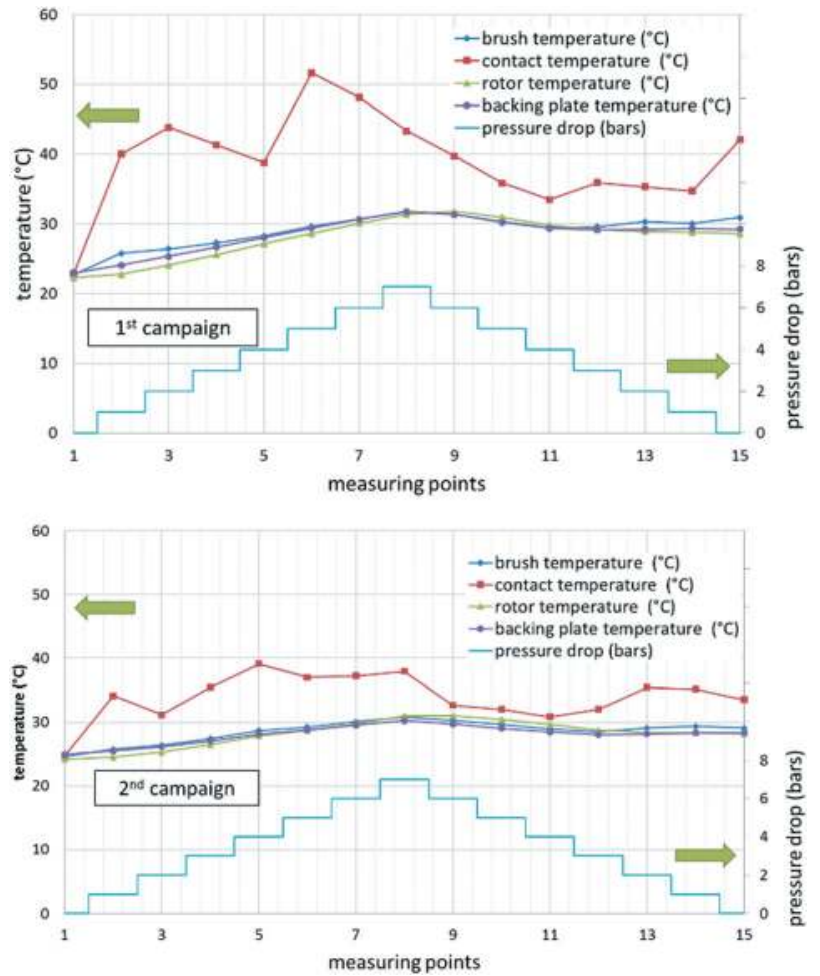

Fig. 16 Temperature variations for $500 \mathrm{~Hz}$ rotation speed (seal with $100 \mu \mathrm{m}$ radial interference)

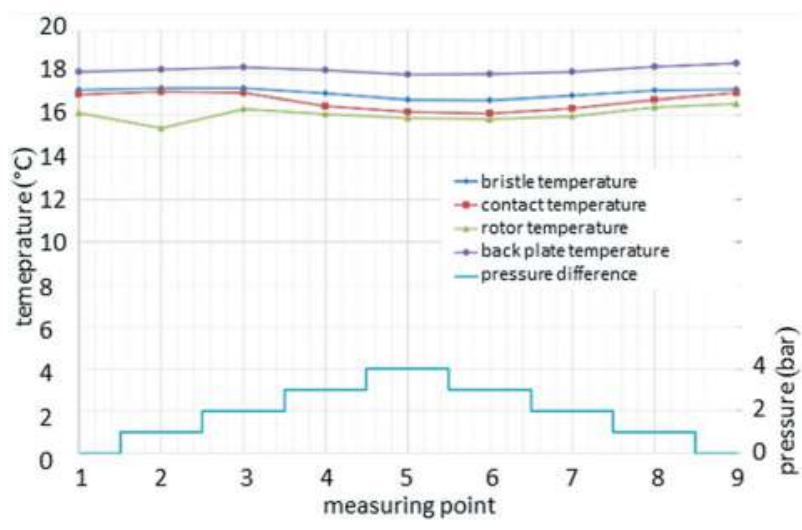

Fig. 17 Temperature variations at $100 \mathrm{~Hz}$ without interference

the rotation speed $(4 \mathrm{bar}$ for $100 \mathrm{~Hz}$ rotation speed and $7 \mathrm{bar}$ for $500 \mathrm{~Hz}$ ). Starting with this point, the temperature of the brush remains constant and may even decrease. This is explained by the fact that the leakage increases with increasing the pressure drop and reaches a point when the convection heat transfer between the bristles and the flow can dissipate the heat generated at their tip. Therefore, the temperature decreases. In the second part of the test, when the pressure difference drops and the leakage flow decreases, the convection heat transfer diminishes and the temperatures increase again.

Finally, only the variations of the contact temperatures are significant, while being quite low (around $5^{\circ} \mathrm{C}$ at $100 \mathrm{~Hz}$ rotation speed and $25^{\circ} \mathrm{C}$ at $500 \mathrm{~Hz}$ ). The brush temperature undergoes only very small variations. This can be explained by the fact that each bristle operates as a heat exchanger fin. As Haynes 25 is a good heat conductor [18] and the leakage flow of the air between the bristles provides a non-negligible convection, the heat generated by the friction of the bristles on the rotor is quickly evacuated. 
Figure 17 depicts the temperatures measured for the seal without radial interference at $100 \mathrm{~Hz}$ rotation frequency. In this case, the temperatures showed almost no variation. The same result was obtained for all the tested rotation speeds proving that when the radial interference is absent, the temperature of the brush seal is not an important parameter.

\section{Comparison With Theoretical Calculations}

The measured flow rates are compared with theoretical results obtained with the model described in Ref. [14]. The theoretical model enables the calculation of the mass flow rate for a given pressure difference. This is based on considering the brush as a deformable, porous medium. The model considers that the rotor is centered, and therefore, only a very small part of the brush shown in Fig. 18 is simulated. This elementary domain is characteristic for the whole seal. It is supposed that the bristles are organized in a hexagonal arrangement and the three rows of bristles enlightened on Fig. 18 represent the minimal requirement for imposing periodicity boundary conditions on the left and on the right-hand side rows of bristles. The unit flow rate obtained for the domain is multiplied with the circumferential length for obtaining the flow rate of the seal.

The geometric characteristics of the elementary domain depicted in Fig. 18 depend on the radius of the bristles and on the bristle density of the brush. This latter piece of information might be subject to interpretation, because the manufacturers report the brush density as a number of bristles per circumferential length of the seal. Reference [14] suggests how the number of bristles in the axial direction can be estimated knowing the brush density. For the tested seals, the bristle diameter is $70 \mu \mathrm{m}$ (Table 1 ). If the bristle pack is supposed completely compact (i.e., zero spacing between bristles), then the number of bristles per $\mathrm{mm}$ in the circumferential direction is roughly 15 . For a brush density of 100 bristles $/ \mathrm{mm}$, an estimated number of 7 bristles in the axial direction is then obtained.

Elastic beams approximate the bristles. The brush is deformed by the pressure gradient and by the contact forces between the

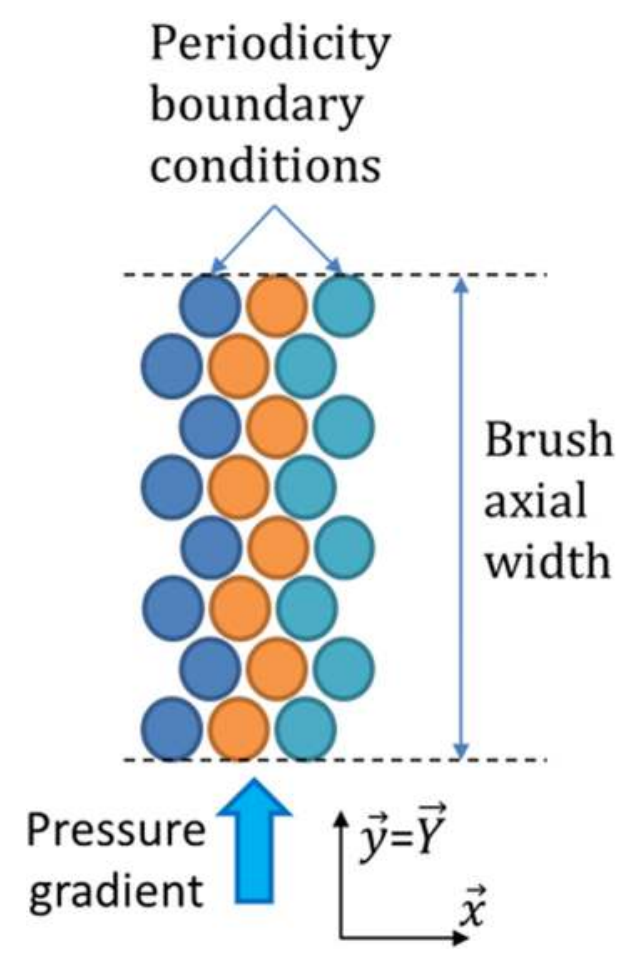

Fig. 18 The simulated brush cell

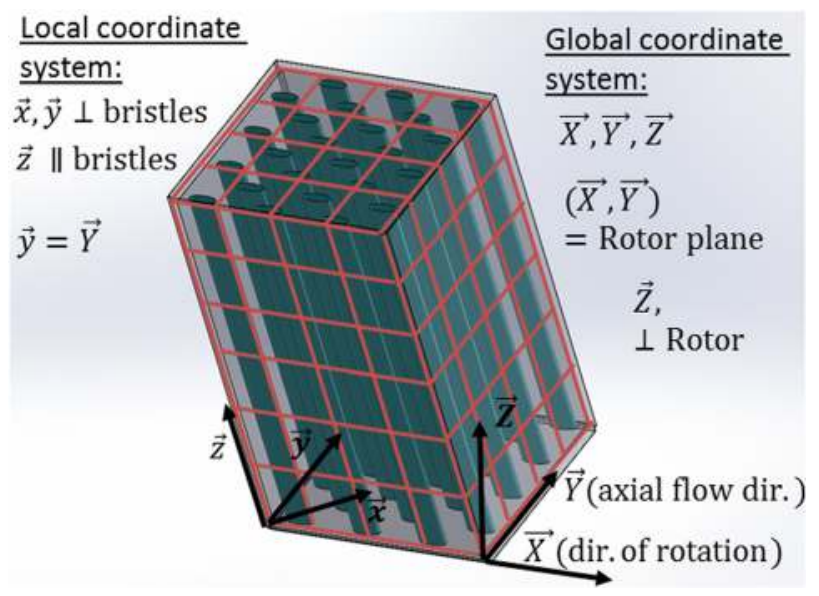

Fig. 19 The computational domain

bristles, the bristles and the rotor, and the bristles and the back plate. The method deals only with brush seals mounted with radial interference; this means that only cases when the bristles are in permanent contact with the rotor can be calculated. This is a simplifying assumption that eliminates the cases with a strong blowdown effect, when the contact between the bristles and the rotor is a priori unknown (open or closed).

The procedure starts by calculating the deformation of the bristles in the absence of the pressure gradient. In this case, the deformation is engendered only by the bristle/rotor and bristle/back plate contacts. The spatially varying porosity is estimated by taking into account the local spacing between the bristles.

The flow in the bristle pack is next calculated by considering the brush a porous medium. Its principal directions in a local coordinate system $\mathbf{x}, \mathbf{y}, \mathbf{z}$ are depicted on Fig. 19. ${ }^{2}$ They are considered to lie along and normal to the undeformed bristles. The flow of air in the porous medium is governed by a generalized elliptic equation having the pressure as unknown. Air is considered a perfect gas and the flow is supposed isothermal. Neglecting energy exchanges inside the brush is a strong simplifying assumption, but its use in the present test cases was encouraged by the limited temperature variations enlightened by measurements. The nonlinear pressure equation writes [14]

$$
\frac{\partial}{\partial x}\left(\frac{K_{x}}{\mu} p \frac{\partial p}{\partial x}\right)+\frac{\partial}{\partial y}\left(\frac{K_{y}}{\mu} p \frac{\partial p}{\partial y}\right)+\frac{\partial}{\partial z}\left(\frac{K_{z}}{\mu} p \frac{\partial p}{\partial z}\right)=0
$$

The spatially varying, anisotropic permeability $K_{i}, i=1,2,3$ was estimated from the similarity between the Darcy-Forheimer equation

$$
-\frac{\Delta P}{\Delta L}=\mu\left(a+\frac{b \rho u}{\mu}\right) u=\frac{\mu}{K} u
$$

and the pressure drop in a duct

$$
\lambda=-\frac{\Delta P / \Delta L}{\rho v^{2} / d_{h}}
$$

A clear distinction should be made between $u$, the filtration velocity in Eq. (2), and $v$, the mean velocity of fluid between bristles. The relation between $u$ and $v$ can be found from the definition of the mass flow rate

\footnotetext{
${ }^{2}$ In Fig. 19 the $\mathbf{y}$ axis of the local coordinate system and the $\mathbf{Y}$ axis of the global coordinate system are parallel and lie in the axial direction. The $\mathbf{z}$ of the local coordinate system is aligned with the bristles while the $\mathbf{Z}$ axis of the global coordinate system is in the radial direction.
} 


$$
q_{m}=S v=S_{f} u \Rightarrow v=\frac{S_{f}}{S} u=c_{v} u
$$

In Ref. [14] the pressure drop was estimated by numerically integrating the time averaged Navier-Stokes equations with a commercial CFD code on three different fluid domains, $\lambda_{i}, i=1$, 2, 3, one for each principal direction of the brush depicted in Fig. 19. Parametric calculations were performed in Ref. [14] for typical porosities and local Reynolds number, $\operatorname{Re}=\rho d_{h} v / \mu$. For the principal directions normal to the bristles, $\mathbf{x}$ and $\mathbf{y}$, the hydraulic diameter was the distance between the bristles. For the principal direction along to the bristles, $\mathbf{z}$, the hydraulic diameter had a more complicated definition given in Ref. [14]. Thus, the theoretical model depends only on the geometric characteristics of the brush (Table 1) and on the operating conditions and is independent of any experimental results

$$
K_{i}(\varepsilon, \operatorname{Re})=\frac{\mu d_{h} u}{\lambda_{i}(\varepsilon, \operatorname{Re}) \rho v^{2}}
$$

Equation (1) requires upstream and downstream pressure boundary conditions in the axial direction. A zero pressure gradient was imposed on the rotor and on the back plate. This corresponds to a zero velocity normal to a solid wall. Periodicity boundary conditions were employed in the circumferential direction.

Considering the brush as a porous medium suggests that the convective inertia effects are neglected, i.e., $b=0$ in Eq. (2). This is only apparent because the pressure drops $\lambda_{i}$ were estimated from full CFD calculations that took into account all terms of the time averaged Navier-Stokes equations. Convective inertia is then intrinsically taken into account by the nonlinear variation of the pressure drop $\lambda_{i}$ with the local Reynolds number. However, consequently of explicitly neglecting convective inertia, velocities cannot be imposed on the boundaries of the computational domain. This means that the solution of the isothermal pressure equation is independent of the rotor speed. ${ }^{3}$

Equation (1) is numerically solved and yields a threedimensional pressure field. New deformations of the bristles are calculated by taking into account the bristle forces applied by the flow pressures and by the bristle/bristle, bristle/rotor, and bristle back/plate contacts. The brush appears then as a three dimensional, deformable porous medium, and the procedure is repeated until convergence. The minimal spacing between the bristles is considered to be of the order of the roughness. A value of $1.2 \mu \mathrm{m}$ was suggested in Ref. [19] and was also used in the present calculations.

Figure 20 depicts the comparison between the experimental results obtained for $100 \mu \mathrm{m}$ radial interference, the numerical mass flow rate calculated with 7 and 8 bristles, and the mass flow rate estimated with the simplified model from Ref. [5]. ${ }^{4}$ The experimental data obtained for different rotation speeds and for the two test campaigns were averaged in order to obtain a leakage mass flow rate depending only on the pressure difference applied to the brush seal.

Globally, the numerically calculated mass flow rate is lower than measurements. However, when the pressure drop increases, the difference becomes smaller and the predictions obtained with 7 bristles correspond to measurements for a pressure difference of $\Delta p=6$ bar. The results obtained with 8 bristles in the axial direction enlighten the sensitivity of the theoretical model to this parameter. The mass flow rates predicted by the simplified model

\footnotetext{
${ }^{3}$ The solution of the porous medium brush flow may depend on the rotor speed if heat transfer is taken into account (i.e., for nonisothermal flow conditions). In that case, the rotor speed multiplied by the friction forces between the bristles and the rotor would provide the heat flux transmitted to the brush.

${ }^{4}$ The results with the simplified model from Ref. [5] are obtained for a value of 0.866 of the parameter $L$ of this model, i.e., for the most compact brush.
}

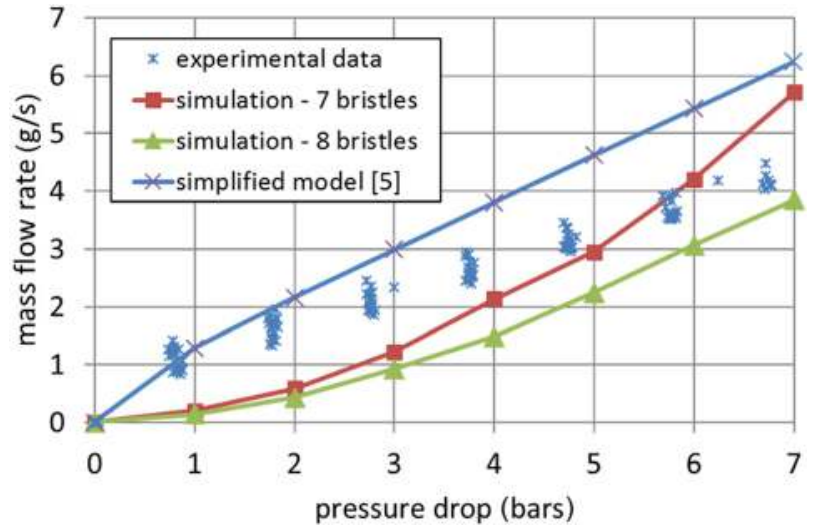

Fig. 20 Comparison between the experimental results obtained with $100 \mu \mathrm{m}$ interference, the numerical calculations realized with 7 and 8 bristles in $y$-direction, and the simplified model from Ref. [5]

from Ref. [5] are somewhat larger and the measurement results lie between the simplified and the numerical approach.

The source of differences between measurement and the predictions is the neglecting of the friction forces in the numerical model. For example, as discussed earlier, the trend in the experiments does not appear to tend linearly to zero mass flow at zero pressure gradient. The increase of the leakage mass flow rate is very rapid for small values of the pressure difference, because the bristles are not compacted yet. The friction forces between bristles prevent the brush from becoming compact. It is only with the increase of the pressure difference that the pressure forces on the bristles will override the friction forces and the brush will become more compact. The same is true for the blow down effect.

Figures 21 and 22 depict the porosity in a section of the brush. As expected, the porosity decreases with increasing the pressure difference. The low values of the porosity are responsible for underpredicting the leakage flow rate for small and mild pressure differences. The average values of the porosities are around 0.15 , while values around 0.2 or 0.3 are used in the literature [10] for adjusting porosity models with experimental data. Low porosities are obtained in the present case because the friction between the bristles in not taken into account. Neglecting bristle friction forces leads to a compactly deformed brush.

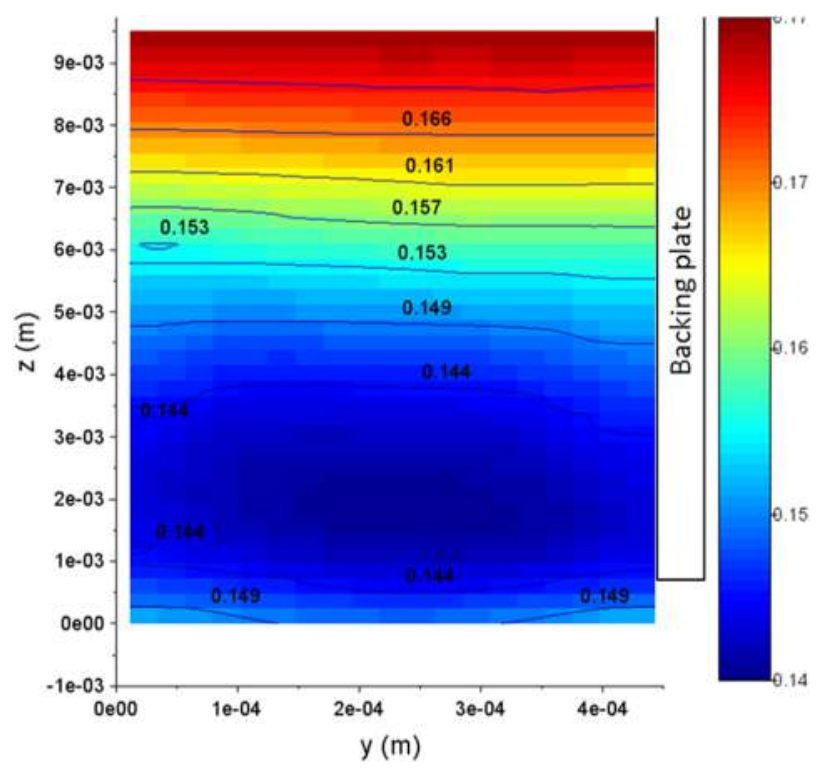

Fig. 21 Porosity field in a section of the brush, $\Delta P=1$ bar, 7 bristles in $y$ direction 


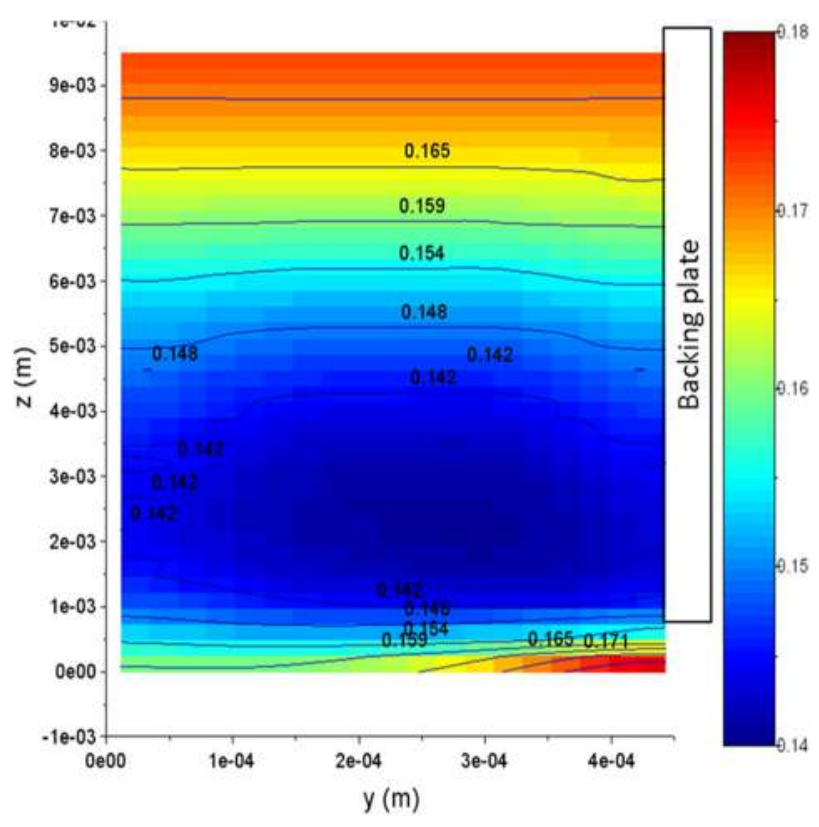

Fig. 22 Porosity field in a section of the brush, $\Delta P=5$ bar, 7 bristles in $y$ direction

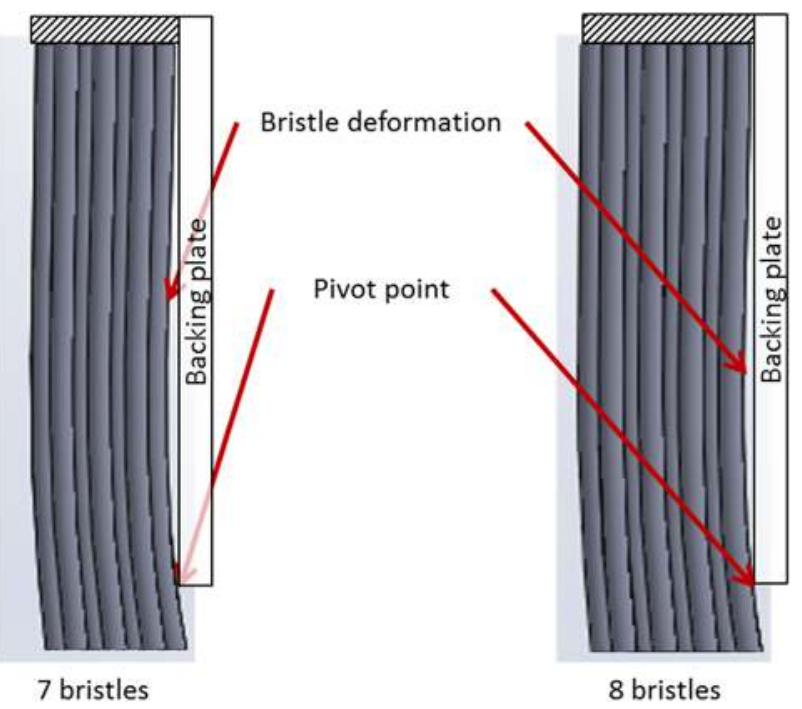

Fig. 23 Deformed brush geometry for $\Delta P=5$ bar

Figure 23 shows the bristles deformation for $\Delta p=5$ bar. The bristles are quite compact and tend to deform strongly only in the zone situated just below the backing plate. This leads to a local rise in the porosity that can be observed also in the lower right corner of Fig. 22. This local increase of the porosity is due to the absence of friction between bristles. Below the backing plate, the bristles do not benefit from any support other than themselves and, if friction forces are neglected, they are free to deform strongly under the influence of the pressure gradient. A similar explanation is given also in Ref. [20]. As $\Delta p$ increases, the strong deformation of the bristles in the zone below the back plate explains why the calculated mass flow rates depicted in Fig. 20 increase faster at higher pressure differences. Furthermore, calculations made with 7 bristles in the axial direction gave porosities below the backing plate that increased quicker with $\Delta p$ than for 8 bristles. A smaller number of bristles provided a weaker support. This explains the steeper increase of the mass flow rate with $\Delta p$ in the case of 7 bristles.

\section{Summary and Conclusions}

The paper presents test results for a brush seal of $38 \mathrm{~mm}$ diameter. This diameter is considered small for usual brush seals and therefore, the running in phase was an important part of the test campaign. The tests provided a useful insight into mass flow variations and operating temperatures.

The first conclusion is that in the present case, the radial interference between the brush seal and the rotor is the leading parameter controlling the leakage flow. Indeed, the mass flow rates obtained without interference are almost twice larger than those with $100 \mu \mathrm{m}$ interference. This indicates that for the tested seals the blow down effect might be limited. Furthermore, the measurements showed quite low temperatures, thus indicating that the metallic brush acts as an effective heat exchanger, dissipating the heat generated by the bristle/rotor contact.

The tests represent an important amount of data available for comparison with theoretical predictions. Calculations performed with a recently published theoretical model gave mass flow rates nearly close to measurements but underpredicted. The advanced explanation is that the low mass flow rates are due to very small porosities. This result is most likely because interbristle, bristle/ backplate, and bristle tip/shaft friction forces are not taken into account in the deformation model of the bristles. Furthermore, the test conditions were not completely free of rotor vibrations and this might have led to an increase of the flow rate that the model cannot take into account at this moment.

\section{Acknowledgment}

The authors are grateful to Centre National d'Etudes Spatiales (CNES) and to Airbus Safran Launchers for supporting this work. They also acknowledge the help of Susan Finding from Faculté des Lettres et Langues of Université de Poitiers in correcting the manuscript.

\section{Nomenclature}

$$
\begin{aligned}
a, b & =\text { coefficients in the Darcy-Forheimer Eq. }(2) \\
c_{v} & =\text { geometrical parameter defined by Eq. }(4) \\
d_{h} & =\text { hydraulic diameter }(\mathrm{m}) \\
K & =\text { permeability }\left(\mathrm{m}^{2}\right) \\
L & =\text { length }(\mathrm{m}) \\
p, P & =\text { pressure }(\mathrm{Pa}) \\
q_{m} & =\text { mass flow rate }(\mathrm{kg} / \mathrm{s}) \\
\mathrm{Re} & =\text { local Reynolds number } \\
S & =\text { surface between bristles }\left(\mathrm{m}^{2}\right) \\
S_{f} & =\text { filtration surface }\left(\mathrm{m}^{2}\right) \\
u & =\text { filtration velocity }(\mathrm{m} / \mathrm{s}) \\
v & =\text { velocity of the fluid between bristles }(\mathrm{m} / \mathrm{s})
\end{aligned}
$$

$\mathbf{x}, \mathbf{y}, \mathbf{z}=$ local coordinate system in Fig. 19

$\mathbf{X}, \mathbf{Y}, \mathbf{Z}=$ global coordinate system in Fig. 19

$\varepsilon=$ local porosity

$\lambda=$ dimensionless pressure drop

$\mu=$ dynamic viscosity $(\mathrm{Pa} \cdot \mathrm{s})$

$\rho=$ is the density $\left(\mathrm{kg} / \mathrm{m}^{3}\right)$

\section{Subscript}

$$
i=\text { principal directions of the brush and bristles }
$$

\section{References}

[1] Hendricks, R. C., Schlumberger, S., Braun, M. J., Choy, F., and Mullen, R. L., 1991, "A Bulk Flow Model of a Brush Seal System," ASME Paper No. 91-GT-325.

[2] Chupp, R. E., Holle, G. F., and Dowler, C. A., 1991, "Simple Leakage Flow Model for Brush Seals," AIAA Paper No. 91-1913.

[3] Chupp, R. E., and Holle, G. F., 1996, "Generalizing Circular Brush Seal Leakage Through a Randomly Distributed Bristle Bed," ASME J. Turbomach., 118(1), pp. 153-161.

[4] Braun, M. J., and Kudriavtsev, V. V., 1995, "A Numerical Simulation of Brush Seal Section and Some Experimental Results," ASME J. Turbomach., 117(1), pp. 190-202.

[5] Chew, J. W., and Hogg, S. I., 1997, "Porosity Modelling of Brush Seals," ASME J. Tribol., 119(4), pp. 769-775. 
[6] Turner, M. T., Chew, J. W., and Long, C. A., 2003, "Experimental Investigation and Mathematical Modeling of Clearance Brush Seals," ASME J. Eng. Gas Turbines Power, 120(3), pp. 573-579.

[7] Dogu, Y., 2005, "Investigation of Brush Seal Flow Characteristics Using Bulk Porous Medium Approach," ASME J. Eng. Gas Turbines Power, 127(1), pp. $136-144$.

[8] Pröstler, S., 2005, "Modellierung Und Numerische Berechnungen Von Wellenabdichtungen in Bürstenbauart," Ph.D dissertation, Bochum Universität, Bochum, Germany.

[9] Guardino, C., and Chew, J. W., 2005, "Numerical Simulation of ThreeDimensional Bristle Bending in Brush Seals," ASME J. Eng. Gas Turbines Power, 127(1), pp. 583-591.

[10] Pugachev, A. O., and Helm, P., 2009, "Calibration of Porous Medium Models for Brush Seals," Proc. Inst. Mech. Eng., Part A, 223(1), pp. 83-91.

[11] Neef, M., Hepermann, F., Sürken, N., and Schettel, J., 2007, "Brush Seal Porosity Modeling-Applicability and Limitations," Seventh European Conference on Turbomachinery, Athens, Greece, Mar. 5-9, Paper No. 110.

[12] Pugachev, A. O., 2013, "Predicted Performance of Brush Seals: Porous Medium Versus Resolved Bristle Matrix and Comparison With Experimental Data," Paper No. ETC2013-054.
[13] Lelli, D., Chew, J. W., and Cooper, P., 2006, "Combined Three-Dimensiona Fluid Dynamics and Mechanical Modeling of Brush Seals," ASME J. Turbomach., 128(1), pp. 188-195.

[14] Deville, L., and Arghir, M., 2018, "Theoretical Analysis of Brush Seal Leakage Using Local CFD Estimated Permeability Laws,” ASME J. Eng. Gas Turbines Power, 140(6), p. 062803

[15] Ergun, S., 1952, "Fluid Flow Through Packed Columns," Chem. Eng. Prog, 48(2), pp. 89-94

[16] Bayley, F. J., and Long, C. A., 1993, "A Combined Experimental and Theoretical Study of Flow and Pressure Distributions in a Brush Seal," ASME J. Turbomach., 115(2), pp. 404-410.

[17] Patentamt, 2000, MTU Motoren- und Turbinen Union München GmbH, Europäaisches Patentnummer 0211275.

[18] Haynes International, 2015, "Haynes 25 Alloy," Haynes International, Inc, Kokomo, IN, accessed Nov. 30, 2016, http://www.haynesintl.com/alloys/ alloy-portfolio_High-temperature-Alloys/haynes-25-alloy

[19] Aksit, M. F., and Tichy, J. A., 1996, "A Computational Model of Brush Seal Bristle Deflection," AIAA Paper No. 96-2909.

[20] Zhao, H., and Stango, R. J., 2007, "Role of Distributed Interbristle Friction Force on Brush Seal Hysteresis," ASME J. Tribol., 129(1), pp. 199-204. 